\title{
Maternidade e HIV: Continuidade do Tratamento e Adesão em Mulheres após Parto
}

\author{
Fernanda Borges de Medeiros' \\ Evelise Rigoni de Faria' \\ Cesar Augusto Piccinini ${ }^{1}$ \\ ${ }^{1}$ Universidade Federal do Rio Grande do Sul (UFRGS), Porto Alegre, Rio Grande do Sul, Brasil
}

\begin{abstract}
Resumo
O estudo examinou fatores sociodemográficos, clínicos e psicossociais que afetam a continuidade do tratamento e a adesão à medicação antirretroviral em mulheres nos primeiros três meses após o parto. Participaram 56 mulheres vivendo com HIV, com idades entre 18 e 43 anos, na sua grande maioria brancas e casadas. Foram utilizadas entrevistas sobre dados sociodemográficos e clínicos, exames laboratoriais e escalas psicológicas. Análises estatísticas revelaram que mais de um terço das participantes $(37,5 \%)$ descontinuaram o próprio tratamento após o parto. A qualidade de vida e o apoio instrumental podem favorecer a continuidade do tratamento de HIV após o parto, e as condições de trabalho e a situação clínica dessas mulheres pode afetar a adesão após o parto. Tais resultados podem contribuir para o desenvolvimento de intervenções que favoreçam a continuidade do tratamento e adesão das mulheres no pós-parto.

Palavras-chave: HIV, gravidez, maternidade, tratamento antirretroviral
\end{abstract}

\section{Motherhood and HIV: Retention in HIV Care and Adherence in Postpartum Women}

\begin{abstract}
This study examined sociodemographic, clinical, and psychosocial factors affecting retention in HIV care and antiretroviral adherence in women during the first three months after delivery. Participants were 56 women living with HIV, aged between 18 and 43 years, mostly white and married. We used interviews about socio-demographic and clinical data, laboratory tests, and psychological scales. Statistical analyses revealed that more than one-third of women $(37.5 \%)$ discontinued their treatment after delivery. Quality of life and instrumental support may contribute to retention in HIV care after childbirth, and the working conditions and clinical status of these women may affect adherence after childbirth. These findings may contribute to the development of interventions that promote retention in HIV care and adherence during the postpartum period. Keywords: HIV; pregnancy; motherhood; antiretroviral treatment.
\end{abstract}

Maternidad y VIH: Continuidad del Tratamiento y Adherencia en Mujeres en el Posparto

Este estudio examinó los aspectos sociodemográficos, clínicos y psicosociales que afectan la continuidad del tratamiento del VIH y adherencia a los antirretrovirales en las mujeres en los tres meses después del parto. Participaron 56 madres infectadas por VIH, con edades comprendidas entre 18 y 43 años. La mayoría eran blancas y estaban casadas. Se utilizaron entrevistas sobre datos sociodemográficos y clínicos, exámenes de laboratorio y escalas psicológicas. Los análisis estadísticos revelaron que más de un tercio de las mujeres (37,5\%) interrumpieron su tratamiento después del parto. La calidad de vida y el apoyo instrumental pueden favorecer la continuidad del tratamiento del VIH después del parto, y que las condiciones laborales y la situación clínica de estas mujeres pueden afectar la adherencia después del parto. Estos resultados pueden contribuir al desarrollo de intervenciones para favorecer la continuidad del tratamiento y la adherencia de las mujeres en el posparto.

Palabras clave: VIH; embarazo; maternidad; tratamiento antirretroviral.

\section{Introdução}

Desde a década de 1990, com o surgimento do tratamento antirretroviral (TARV), a Síndrome da Imunodeficiência Adquirida (AIDS), causada pelo vírus da imunodeficiência humana (HIV), tem sido considerada uma doença crônica, em função da melhoria da qualidade de vida e das condições de saúde das pessoas que vivem com HIV em uso de TARV. O uso correto dessas medicações tem possibilitado suprimir a replicação do vírus e restaurar parcialmente o sistema imunológico das pessoas que vivem com HIV, levando à redução das complicações relacionadas à AIDS (Deeks, Lewin, \& Havlir, 2013; Grinsztejn et al., 2014; Lundgren et al.,
2015). Além da melhora da saúde, o TARV reduz consideravelmente a transmissão do vírus e prolonga os anos de vida dos pacientes (Cohen et al., 2011; Deeks et al., 2013; Lundgren et al., 2015).

O início de TARV de forma precoce logo após o diagnóstico, independentemente de condições clínicas, tem se mostrado benéfico para a saúde das pessoas que vivem com HIV. Entretanto, a adesão à medicação antirretroviral tem sido um dos principais desafios no cuidado de pessoas vivendo com HIV no mundo e no Brasil. Especificamente, entre as mulheres, foco do presente estudo, tem se demonstrado que a adesão à medicação antirretroviral após o parto geralmente diminui (Henegar et al., 2015; Kreitchmann et al., 2012; 
Mellins et al., 2008; Nachega et al., 2012). Do mesmo modo, logo após o parto, as taxas de continuidade do tratamento do $\mathrm{HIV}^{1}$ em mulheres também são bastante reduzidas (Adams, Brady, Michael, Yehia, \& Momplaisir, 2015; Buchberg et al., 2015; Phillips et al., 2014).

Para Organização Mundial de Saúde - OMS (World Health Organization [WHO], 2015), o método mais confiável para medir a adesão é o nível de supressão viral. Já, na avaliação da continuidade do tratamento, ainda não existe um consenso (Kay, Batey, \& Mugavero, 2016). Por não ser uma medida dicotômica, como discutido por Mugavero et al. (2012), a avaliação da continuidade do tratamento é bastante complexa, já que envolve múltiplas visitas, agendadas em intervalos de tempo variáveis e que ocorrem ao longo do tempo. Por toda essa complexidade, esses autores sugerem que a sua avaliação seja adaptada ao contexto de investigação.

A continuidade do tratamento está relacionada à supressão viral, possibilitando diminuir risco de progressão da doença (Momplaisir, Storm, Nkwihoreze, Jayeola, \& Jemmott, 2018). Tem se evidenciado que a descontinuidade do tratamento de HIV leva ao aumento significativo de carga viral (CV) e diminuição da contagem de linfócito TCD4 (Aebi-Popp et al., 2016), aumentando o risco de mortalidade das pessoas que vivem com HIV (Mugavero et al., 2014).

Por toda sua importância para a saúde das mulheres, tem-se buscado avaliar e compreender fatores que favorecem a continuidade do tratamento de HIV e a adesão à TARV. Características sociodemográficas têm sido associadas à continuidade do tratamento em mulheres após o parto. Em alguns estudos, as mulheres negras e com menor idade mais frequentemente não compareciam a suas consultas médicas após o parto (Siddiqui, Bell, Sangi-Haghpeykar, Minard, \& Levison, 2014; Tariq et al., 2016). Como discutido por Tariq et al. (2016), possivelmente, as mulheres negras residentes na Inglaterra, que eram migrantes, enfrentavam muitos desafios para continuidade do tratamento, incluindo desde pobreza, dificuldades de acesso à medicação e isolamento social. De fato, a falta de apoio social é uma das barreiras, que leva as mulheres a descontinuarem o próprio tratamento (Buchberg et al., 2015). Geralmente, está relacionada ao estigma já vivido ao revelar o diagnóstico. Por isso, ao não expor sua sorologia para

\footnotetext{
Em inglês, o termo utilizado é "retention in HIV care". Em português, poderia ser traduzido por retenção ou continuidade do tratamento de HIV. Contudo, optou-se por traduzir para o português como "continuidade do tratamento", termo utilizado no presente estudo.
}

pessoas da família, geralmente a rede de apoio social delas é bastante limitada, dificultando que possam ser auxiliadas nas diversas tarefas que incluem trabalho, escola de filhos e os cuidados com sua saúde e a do bebê (Buchberg et al., 2015).

Além desses fatores interpessoais e sociodemográficos, a literatura tem demonstrado que mulheres diagnosticadas durante a gestação são mais propensas a abandonar o tratamento (Adams et al., 2015; Phillips et al., 2014). Do mesmo modo, CV detectável na gestação e início tardio do pré-natal e de TARV também têm sido associados à descontinuidade do tratamento após o parto (Adams et al., 2015; Phillips et al., 2014; Swain et al., 2016). Geralmente, essas mulheres com início tardio das consultas do pré-natal têm pouco tempo para serem aconselhadas sobre o tratamento. Em contrapartida, no que tange ao estágio da infecção pelo HIV, tem-se evidenciado que aquelas com melhores condições clínicas, com contagem de TCD4 acima de 350 células $/ \mathrm{ml}$, mais frequentemente abandonaram o próprio tratamento (Tariq et al., 2016). Diferentemente da gestação em que a motivação para tratamento é a responsabilidade com o bebê, após o parto, a continuidade dele dependerá da noção de autocuidado da mulher (Siddiqui et al., 2014) e da preocupação com sua própria saúde (Kempf et al., 2010).

Similarmente, a literatura tem identificado que existe uma tendência de as mulheres seguirem mais frequentemente a medicação durante a gestação, provavelmente pela preocupação com a prevenção da transmissão vertical do vírus e com a saúde do bebê (Faria \& Piccinini, 2015; Nachega et al., 2012; Ngnarina et al., 2013). Além da preocupação com a saúde do feto, o fato das mulheres grávidas frequentarem com mais regularidade os serviços de saúde parece contribuir para a adesão delas na gestação (Faria et al, 2014; Hodgson et al., 2014). Isso propiciaria oportunidades de aconselhamento, apoio emocional e acesso a informações, podendo favorecer a adesão durante a gestação. Nesse sentido, na gestação, assim como ocorre com os serviços e profissionais de saúde que dirigem sua atenção para os cuidados da saúde do bebê, a principal preocupação da mulher é proteger a criança, evitando a transmissão vertical (Faria \& Piccinini, 2015; Ngnarina et al., 2013), o que diminui a motivação delas para continuar a medicação depois do nascimento da criança.

Além disso, tem-se apontado que condições adversas de vida podem dificultar a adesão das mulheres após o parto. Dentre essas, destacam-se o uso de álcool, a depressão pós-parto, o baixo nível socioeconômico e a 
baixa escolaridade (El-Khatib et al., 2011; Kreitchmann et al., 2012; Nachega et al., 2012). Somam-se a isso, outras características sociodemográficas, como idade e raça, sendo que as mulheres negras e as mais jovens tendem a apresentar menor adesão (Hodgson et al., 2014; Mellins et al., 2008). Todas essas características podem impor barreiras de acesso aos serviços de saúde, impossibilitando a adesão dessas mulheres. Do mesmo modo, no estudo de Mellins et al. (2008), aquelas com piores condições de saúde física - com maior número e maior gravidade de sintomas físicos - assim como aquelas com CV detectável durante a gestação, foram as que apresentaram pior adesão após o parto.

Por fim, diversos estudos têm demonstrado que, após o parto, o suporte social é outro preditor da adesão em mulheres (El-Khatib et al., 2011; Gugsa et al., 2017; Hodgson et al., 2014), incluindo o suporte financeiro, instrumental e emocional. Esses pesquisadores também ressaltaram a importância da revelação do diagnóstico para um familiar, o parceiro ou outra pessoa significativa, na medida em que isso facilita que a mulher possa receber diversas formas de ajuda, seja para buscar a medicação, apaziguar os medos suscitados pelo tratamento e/ou auxílio financeiro, contribuindo para continuar o uso da medicação, mesmo após o nascimento do filho.

Como visto anteriormente, entre as mulheres, é frequente o abandono do próprio tratamento após o parto. Trata-se de um fenômeno bastante complexo e multifatorial. Embora muitos estudos já tenham demonstrado a influência de fatores sociodemográficos, clínicos e psicossociais na continuidade do tratamento e adesão em mulheres após o parto (Adams et al., 2015; Buchberg et al., 2015; Kreitchmann et al., 2012; Mellins et al., 2008; Nachega et al., 2012; Phillips et al., 2014; Swain et al., 2016), no Brasil, ainda são escassos os estudos que enfocam a adesão entre as mulheres após o parto (Kreitchmann et al., 2012), sendo que não foram encontradas investigações sobre continuidade do tratamento em mulheres vivendo com HIV. Sendo assim, o presente estudo buscou examinar os fatores sociodemográficos, clínicos e psicossociais que afetam a continuidade do tratamento e a adesão à medicação antirretroviral em mulheres nos primeiros três meses após o parto.

Levando em consideração os estudos revisados, esperava-se encontrar menor continuidade do tratamento entre mulheres com início tardio do pré-natal, diagnóstico recente e CV detectável na gestação. Também era esperado que mulheres com mais idade e suporte social tendessem a continuar tratamento após o parto. Já, quanto à adesão no terceiro mês após o parto, esperava-se encontrar menor adesão entre as mulheres mais jovens, com menor apoio social, com CV detectável no terceiro trimestre da gestação e com depressão.

\section{Método}

\section{Participantes}

Participaram 56 mulheres vivendo com HIV, com idades entre 18 e 43 anos $(M=27,88 ; D P=6,07)$ e que foram acompanhadas do último trimestre da gestação ao terceiro mês de vida do bebê. A maioria das participantes se autodeclarou branca (64\%) e estava casada ou coabitava com parceiro fixo (73\%). Quanto à escolaridade, a maior parte da amostra apresentava baixa escolaridade (57\%), com 0 a 8 anos de estudo concluídos; e 36\%, com 9 a 11 anos de estudo. Aproximadamente metade delas (49\%) estava trabalhando, e a maioria já tinha outros filhos $(86 \%)$. A maior parte recebeu o diagnóstico da infecção pelo HIV antes da gestação atual $(78 \%)$ e já tinha revelado o diagnóstico para o pai do bebê $(93 \%)$ e/ou para família (80\%). Um terço delas $(32 \%)$ tinha recebido o diagnóstico recentemente nos últimos dois anos, antes de sua participação nesta pesquisa, e muitas já tinham vivenciado outra gestação com HIV (43\%). Quase metade das participantes (43\%) vivia com um parceiro com HIV.

No que se refere à situação clínica, a maioria das mulheres (55\%) apresentou contagem de TCD4 superior a 350 células/ $\mathrm{ml}$ no primeiro exame realizado durante a gestação (em sua maioria no primeiro trimestre). Quanto à adesão à medicação antirretroviral durante a gestação, a maioria (62\%) estava com CV indetectável (CV $\leq$ 50 cópias $/ \mathrm{ml}$ ) no terceiro trimestre, indicando adesão adequada. Após o parto, a maioria delas (64\%) deveria continuar com TARV. Quanto à saúde mental, quase um terço $(27 \%)$ apresentou diagnóstico de depressão moderada ou grave (BDI $\geq 20)$ no $3^{\circ}$ mês de vida do bebê, enquanto poucas (18\%) relataram uso abusivo de álcool e/ou outras drogas na vida. Por fim, quanto à transmissão vertical, dentre os bebês $(71 \%)$ que realizaram exame de $\mathrm{CV}$ durante o primeiro ano de vida, todos apresentaram resultado não reagente.

As participantes do presente estudo foram selecionadas de projeto maior "Aspectos psicossociais, adesão ao tratamento e saúde da mulher no contexto do HIV/AIDS: Contribuições de um programa de intervenção da gestação ao segundo ano de vida do bebê, 2005-2011" - PSICAIDS - que acompanhou gestantes 
em um centro de referência para HIV em Porto Alegre, detalhado na tese da primeira autora ${ }^{2}$. O projeto foi aprovado pelo Conselho de Ética da Universidade Federal do Rio Grande do Sul e do hospital onde foi realizado. O PSICAIDS envolveu três estudos. O Estudo 1 teve a participação de 64 gestantes, acompanhadas na gestação e no $3^{\circ}, 12^{\circ}$ e $24^{\circ}$ mês de vida do bebê. Já, o Estudo 2 envolveu 26 gestantes que participaram de uma intervenção psicoeducativa durante a gestação e que foram acompanhadas no pós-intervenção, no $3^{\circ} \mathrm{e}$ $12^{\circ}$ mês de vida do bebê. Em ambos os estudos, os critérios de inclusão eram: gestante com diagnóstico de infecção pelo HIV, ter gestação de feto único, ter 18 anos ou mais, não ser usuária de drogas e não apresentar debilidade física severa ou distúrbios mentais. Por fim, o Estudo 3 incluiu 68 que integraram os Estudos 1 e 2 e que realizaram exames laboratoriais na gestação e após parto para investigar os subtipos do HIV presentes e o impacto da profilaxia da transmissão vertical na biologia do vírus.

Para fins deste estudo, foram considerados os dados coletados no Estudo 1 e 2 do PSICAIDS e incluídos os casos que participaram da coleta de dados no último trimestre da gestação e no $3^{\circ}$ mês de vida do bebê. Dentre as participantes do Estudo 1, 35 mulheres realizaram as coletas de dados da Fase I (Gestação) e da Fase II ( $3^{\circ}$ mês); e do Estudo 2, 21 mulheres. Sendo assim, foram excluídas aquelas $(N=34)^{3}$ que não participaram da coleta de dados da Fase II ( $3^{\circ}$ mês), totalizando 29 participantes (45\%) do Estudo 1 e cinco participantes $(19 \%)$ do Estudo 2. Na maioria das variáveis sociodemográficas e clínicas, as 34 mulheres que não realizaram a coleta de dados da Fase II ( $3^{\circ}$ mês) não apresentaram diferenças significativas das participantes do presente estudo, exceto quanto à escolaridade e à idade gestacional de início do pré-natal. Aquelas que não realizaram as coletas de dados do $3^{\circ}$ mês eram menos escolarizadas ( $M=6$ anos de estudo) e com início mais tardio do pré-natal ( $M=16$ semanas de gestação).

Cabe destacar que, para as análises sobre continuidade do tratamento nos três meses após o parto, foram incluídas 56 participantes. Já, para as análises da

\footnotetext{
2 Medeiros, F. B. (2018). Maternidade e HIV: Retenção e Adesão ao Tratamento da Gestação ao Terceiro Mês do Bebê (Tese de doutorado). Universidade Federal do Rio Grande do Sul.

3 Dentre essas mulheres que não realizaram a coleta de dados na Fase II ( $3^{\circ}$ mês), 30 desistiram de participar do estudo ou não puderam ser contatadas no $3^{\circ}$ mês; três delas perderam o bebê (um aborto, um natimorto e um bebê falecido nas primeiras 24 horas de vida); e uma delas estava presa.
}

adesão à medicação antirretroviral nos três meses após o parto, foram considerados os casos de mulheres $(n=$ 24) vivendo com $\mathrm{AIDS}^{4}$ e que realizaram a coleta de $\mathrm{CV}$ durante a Fase II ( $3^{\circ}$ mês).

\section{Instrumentos}

Entrevista sobre Dados Sociodemográficos da Família. Entrevista estruturada com objetivo de investigar dados sociodemográficos da mulher e de sua família e informações relativas à infecção pelo HIV e tratamento.

Protocolo de avaliação da adesão ao tratamento para HIV / AIDS. Esse protocolo reuniu informações sobre o diagnóstico, a saúde e o tratamento da mãe, bem como sobre a profilaxia da transmissão vertical. Esses dados eram perguntados diretamente à gestante e complementados com informações de prontuário.

Escala de Suporte Social para Pessoas Vivendo com HIV / AIDS (Seidl \& Tróccoli, 2006). Adaptada à população brasileira por Seidl e Tróccoli (2006). A análise fatorial realizada pelos autores resultou numa solução de dois fatores: suporte emocional (12 itens; $\alpha=$ $0,92)$ e suporte instrumental (12 itens; $\alpha=0,84$ ). Os dois fatores apresentaram uma correlação de 0,57 entre si. A escala é composta de 24 itens que avaliam duas dimensões de apoio social (instrumental e emocional), considerando-se o apoio recebido, a satisfação com o apoio e a fonte de apoio $(\alpha=0,87)$.

Questionário sobre a Qualidade de Vida de Pessoas Portadoras do HIV - WHOQoL-HIV-Bref (Zimpel \& Fleck, 2007). Trata-se de um instrumento de avaliação de qualidade de vida (QV) de pessoas que vivem com HIV, construído pela OMS, validado e adaptado para a população brasileira por Zimpel e Fleck (2007). Possui 31 perguntas, das quais cinco questões são específicas sobre a infecção pelo HIV. É composto por seis domínios, sendo que cada um inclui um conjunto de facetas. O primeiro domínio do questionário, Físico, inclui as facetas: dor e desconforto, energia e fadiga, sono e descanso, e sintomas referentes à infecção pelo HIV $(\alpha=0,88)$. O segundo domínio, Psicológico, é composto pelas facetas: sentimentos positivos, sentimentos negativos, cognição (concentração), corpo (aparência), autoestima $(\alpha=0,93)$. O terceiro domínio, Nível de Independência, é composto pelas facetas: mobilidade, atividades de vida diária, dependência de medicação ou tratamentos e aptidão ao trabalho $(\alpha=0,91)$. O quarto domínio, Relações Sociais, inclui as facetas:

4 No período em que PSICAIDS foi conduzido (2005-2011), TARV era suspenso após o parto em mulheres assintomáticas e com TCD4 menor que 200 células $/ \mathrm{mm}^{3}$. 
relacionamentos interpessoais, apoio social, atividade sexual e inclusão social $(\alpha=0,87)$. O quinto domínio, Ambiente, é composto pelas facetas: segurança física, moradia, finanças, cuidados (acesso e qualidade à saúde), informações (adquirir informação nova/aprender novas habilidades), lazer, ambiente físico (poluição, barulho, trânsito, clima) e transporte $(\alpha=0,90)$. O sexto domínio, Espiritualidade/Religiosidade/Crenças Pessoais, inclui as facetas de perdão e culpa, preocupações sobre o futuro, morte e morrer $(\alpha=0,87)$.

Inventário Beck de Depressão - BDI (Cunha, 2001). Avaliou presença e gravidade de indicadores de depressão. Trata-se de uma escala para avaliação de sintomas depressivos no formato de autorrelato, composta por 21 itens com alternativas de resposta sobre como a pessoa está se sentindo durante a última semana, incluindo o dia da aplicação. As respostas correspondem a diferentes níveis de gravidade de depressão: mínimo (até 11 pontos), leve (de 12 a 19 pontos), moderado (de 20 a 35 pontos) ou grave (acima de 36 pontos). O estudo de validação do BDI para a população brasileira (Cunha, 2001) apresentou uma consistência interna de 0,84 e a correlação entre teste e reteste foi de $0,4018(p<0,001)$.

\section{Procedimentos}

Utilizou-se um delineamento longitudinal (Robson \& McCartan, 2016) para examinar os fatores sociodemográficos, clínicos e psicossociais que afetam a continuidade do tratamento e a adesão à medicação antirretroviral em mulheres nos primeiros três meses após o parto. A continuidade do tratamento foi definida a partir da realização da coleta de exame de CV e TCD4 durante os três meses após o parto. Já, a adesão à medicação antirretroviral foi definida como contagem de $\mathrm{CV}$ indetectável $(\leq 50$ cópias $/ \mathrm{ml}$ ) nos três meses após o parto.

As participantes foram inicialmente contatadas no serviço de saúde no qual realizavam seu acompanhamento pré-natal e convidadas a participar do estudo. Aquelas que aceitaram, assinaram o Termo de Consentimento Livre e Esclarecido e responderam à Entrevista sobre Dados Sociodemográficos da Família. Quando estavam no terceiro trimestre da gestação, foi agendado um encontro para a coleta de dados da Fase I, quando responderam ao Protocolo de avaliação da adesão ao tratamento para HIV/AIDS. Também foi aplicada a Escala de Suporte Social para Pessoas Vivendo com HIV/AIDS e o WHOQoL-HIV-Bref. Na Fase II, no terceiro mês de vida do bebê, além dos instrumentos da Fase I, as participantes também responderam ao BDI.

\section{Análise de Dados}

Para fins de análise dos dados, foi utilizado o software SPSS 17.0. Inicialmente, foi conduzida uma análise descritiva dos dados e verificada a normalidade dos dados por meio do teste de Kolmogorov-Smirnov. No caso de distribuição normal, foi utilizado o teste $t$ para amostras independentes a fim de avaliar diferenças entre os grupos; e teste Mann-Whitney, para as variáveis sem distribuição normal. $\mathrm{Na}$ análise de variáveis categóricas, foi utilizado o teste Qui-quadrado ou teste exato de Fisher.

\section{Resultados}

Com relação à continuidade do tratamento nos três meses após o parto, 35 (62,5\%) realizaram coleta do exame de exame de CV e/ou TCD4. Foram analisadas as diferenças entre as mulheres que realizaram uma coleta de exame nos três meses após o parto (Grupo 1: com coleta de exame) ou que não o fizeram (Grupo 2: sem coleta de exame). No que se refere às variáveis sociodemográficas, não foram encontradas diferenças significativas. Contudo, uma diferença marginalmente significativa apareceu associada ao trabalho da mulher. Comparadas às mulheres que não trabalhavam, as que estavam trabalhando tenderam a apresentar mais continuidade do tratamento $\left(X^{2}=3,37, p=0,07\right)$.

Quanto às variáveis clínicas, os resultados indicaram diferenças significativas da primeira contagem de TCD4 da gestação $(t(50)=2,28, p=0,03)$, com tamanho de efeito intermediário $(d=0,66, r=0,29)$. As mulheres que realizaram a coleta de exame nos três meses após o parto apresentaram menor contagem de TCD4 gestação $(M=367)$ em comparação às que não realizaram coleta nos três meses após o parto $(M$ $=511)$. Não houve diferenças significativas para as demais variáveis. A Tabela 1 apresenta as características sociodemográficas, clínicas e obstétricas das participantes por grupo, de acordo com continuidade ou não do tratamento.

Já, quanto às características psicossociais, verificou-se diferenças significativas entre o Grupo 1 (com continuidade) e Grupo 2 (sem continuidade), quanto ao apoio instrumental percebido pelas mães no $3^{\circ}$ mês de vida do bebê $(t(53)=2,39 ; p=0,02)$, com $d=0,67$ $(I C=1,22-0,11), r=0,29$, tamanho de efeito intermediário. Aquelas que realizaram coleta do exame no $3^{\circ}$ mês (Grupo 1 com continuidade) apresentaram maiores escores de apoio instrumental $(M=3,78)$ do que as do Grupo 2, que não realizaram $(M=3,25)$. Cabe 
Tabela 1.

Características Sociodemográficas, Clínicas e Obstétricas das Participantes, de Acordo com Continuidade ou Não do Tratamento

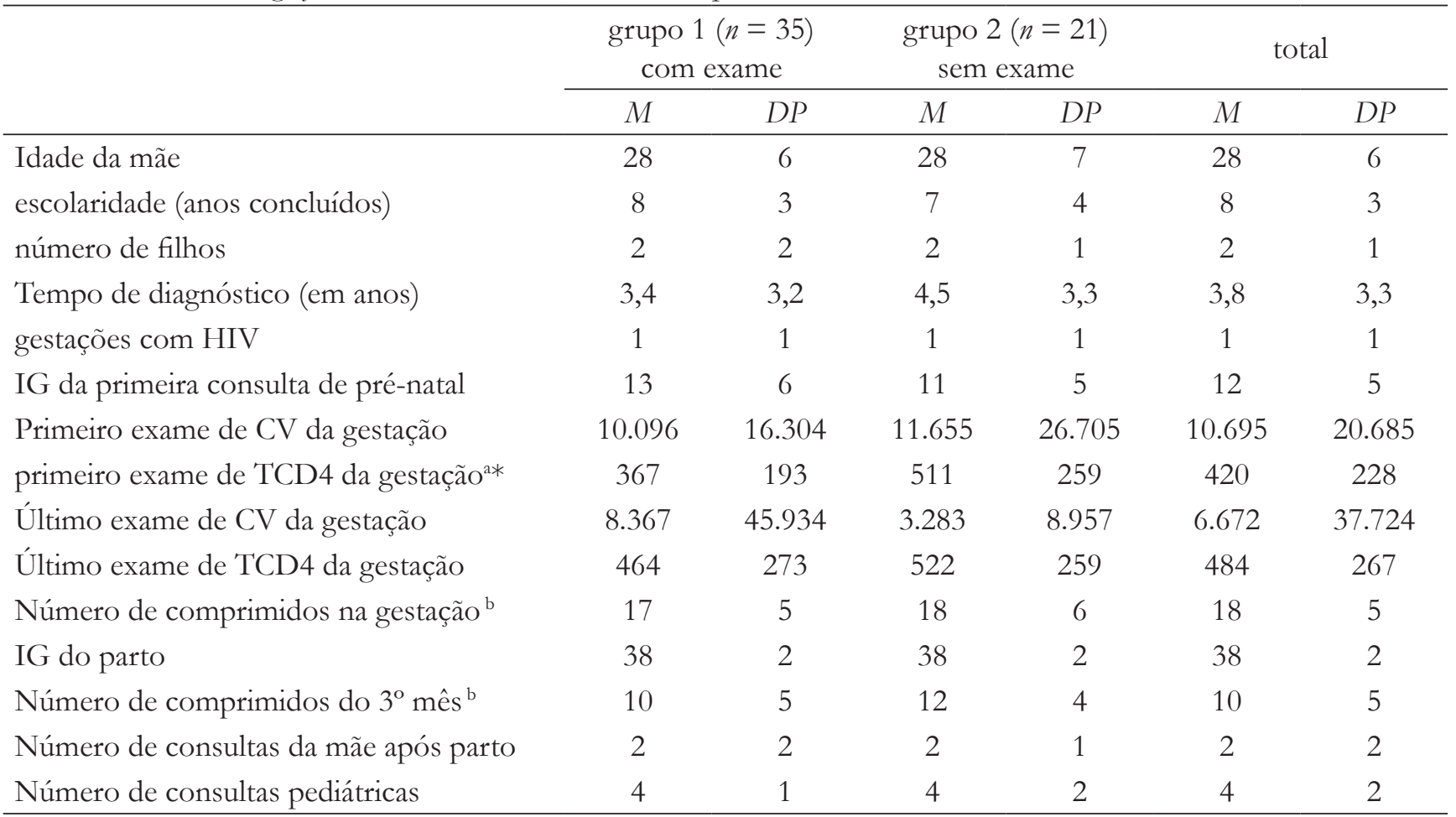

também destacar que houve diferença significativa quanto ao escore de qualidade de vida no domínio do Ambiente durante a gestação entre os dois grupos $(U$ $=215,000 ; p=0,02)$, com tamanho de efeito intermediário $\left(d=0,64, r=0,29 ; \eta^{2}=0,09\right)$. O Grupo 1 (com continuidade) apresentou maior média nos escores de qualidade de vida no domínio do Ambiente, na gestação, do que o Grupo 2 (sem continuidade). A Tabela 2 apresenta as características psicossociais das participantes, por grupo de acordo com continuidade ou não do tratamento.

Também foi utilizado teste Qui-quadrado para avaliar as variáveis categóricas. Contudo, não foram encontradas diferenças significativas quanto à situação conjugal, raça, momento do diagnóstico, CV detectável no terceiro trimestre da gestação, efeitos colaterais, diagnóstico do pai do bebê e revelação do diagnóstico.

No que se refere à adesão, como já mencionado, foi avaliada a partir da coleta de exame de CV nos três meses após o parto. Dentre as 36 mulheres com indicação de seguir medicação antirretroviral, 66\% delas realizaram a coleta de CV após o parto $(n=24)$, sendo que destas apenas a metade apresentou CV indetectável $(\leq 50$ cópias $/ \mathrm{ml})$ nos três meses após o parto.
Na comparação entre o Grupo 1 (com adesão) e Grupo 2 (sem adesão), utilizando o teste exato de Fisher, identificou-se que a grande maioria das mulheres que trabalhavam (71\%) apresentou CV indetectável nos três meses após o parto $(\phi=0,03)$. Quanto às demais variáveis sociodemográficas, não foram encontradas diferenças significativas. No que se refere às características clínicas, verificou-se diferenças significativas entre os dois grupos no resultado da última contagem de TCD4 da gestação $(t(17)=2,36$; $p=0,03)$. O Grupo 1, com adesão após o parto, havia apresentado maior contagem de TCD4 na gestação $(M=433,90)$ do que as do Grupo 2 , sem adesão $(M$ $=236,89)$, com grande tamanho de efeito $(d=1,08$; $r=0,45)$. Da mesma forma, a média da contagem de TCD 4 do $3^{\circ}$ mês foi maior entre o Grupo 1 , de mulheres com adesão $(t(21)=2,28 ; p=0,03)$, com grande tamanho de efeito $(d=0,95 ; r=0,41)$, do que no Grupo 2, sem adesão. Ainda no que se refere às características clínicas das mulheres, utilizando o teste exato de Fisher, a análise de dados revelou que, com exceção de uma participante, todas demais mulheres do Grupo 1, com adesão, já estavam com CV indetectável no último trimestre da gestação $(p=0,04)$. 
Tabela 2.

Características Psicossociais das Participantes, de Acordo com Continuidade ou Não do Tratamento

\begin{tabular}{|c|c|c|c|c|c|c|c|c|}
\hline & \multicolumn{2}{|c|}{$\begin{array}{c}\text { grupo } 1(n=35) \\
\text { com exame }\end{array}$} & \multicolumn{2}{|c|}{$\begin{array}{c}\text { grupo } 2(n=21) \\
\text { sem exame }\end{array}$} & \multicolumn{4}{|c|}{ total } \\
\hline & $M$ & $D P$ & $M$ & $D P$ & M & $D P$ & mín. & máx. \\
\hline \multicolumn{9}{|l|}{ Gestação } \\
\hline \multicolumn{9}{|l|}{ Apoio } \\
\hline Total & 3,75 &, 72 & 3,49 &, 78 & 3,65 &, 75 & 2,05 & 4,85 \\
\hline Instrumental & 3,84 &, 72 & 3,50 & ,89 & 3,72 &, 79 & 1,80 & 5,00 \\
\hline Emocional & 3,67 & ,93 & 3,50 &, 80 & 3,61 &, 88 & 1,70 & 5,00 \\
\hline \multicolumn{9}{|l|}{ QV } \\
\hline Físico & 12,88 & 3,50 & 12,58 & 3,27 & 12,77 & 3,39 & 6,00 & 20,00 \\
\hline Psicológico & 13,53 & 3,18 & 13,14 & 2,99 & 13,39 & 3,09 & 4,80 & 19,20 \\
\hline Independência & 12,09 & 3,25 & 12,45 & 3,03 & 12,22 & 3,15 & 5,00 & 19,00 \\
\hline Relações sociais & 14,56 & 2,81 & 13,58 & 3,04 & 14,21 & 2,90 & 7,00 & 20,00 \\
\hline Ambiente ${ }^{a *}$ & 14,26 & 8,58 & 11,58 & 2,73 & 13,27 & 7,09 & 6,00 & 61,00 \\
\hline Espiritualidade & 13,52 & 2,64 & 14,42 & 3,55 & 13,85 & 3,00 & 8,00 & 20,00 \\
\hline Total & 80,82 & 14,65 & 77,79 & 14,72 & 79,75 & 14,60 & 49,80 & 122,20 \\
\hline \multicolumn{9}{|l|}{$3^{\circ}$ mês } \\
\hline \multicolumn{9}{|l|}{ Apoio } \\
\hline Total & 3,68 & ,67 & 3,32 &, 78 & 3,55 &, 73 & 1,80 & 4,85 \\
\hline Instrumental $^{\mathrm{b} *}$ & 3,78 & ,69 & 3,25 & 94 & 3,59 &, 82 & 1,30 & 5,00 \\
\hline Emocional & 3,58 & ,86 & 3,39 &, 84 & 3,51 &, 85 & 1,60 & 5,00 \\
\hline \multicolumn{9}{|l|}{ QV } \\
\hline Físico* & 15,17 & 3,00 & 13,45 & 3,19 & 14,55 & 3,16 & 8,00 & 20,00 \\
\hline Psicológico & 13,78 & 3,11 & 12,60 & 3,33 & 13,35 & 3,21 & 7,20 & 20,00 \\
\hline Independência & 14,37 & 3,16 & 13,11 & 2,75 & 13,93 & 3,06 & 6,00 & 20,00 \\
\hline Relações sociais & 14,40 & 2,74 & 14,20 & 2,75 & 14,33 & 2,72 & 6,00 & 20,00 \\
\hline Ambiente & 13,16 & 2,62 & 11,79 & 2,58 & 12,68 & 2,66 & 7,00 & 18,50 \\
\hline Espiritualidade & 13,89 & 2,75 & 15,10 & 3,49 & 14,33 & 3,07 & 9,00 & 20,00 \\
\hline Total & 84,77 & 12,78 & 80,45 & 14,47 & 83,25 & 13,43 & 46,20 & 111,70 \\
\hline depressão ${ }^{c}$ & 14 & 9 & 18 & 14 & 15 & 11 & 0 & 42 \\
\hline
\end{tabular}

$M=$ média; $D P=$ desvio padrão; mín = mínimo; máx = máximo; $\mathrm{QV}=$ qualidade de vida.

${ }^{a}$ teste Mann-Whitney.

b teste $t$.

${ }^{c}$ escore total no inventário Beck de depressão.

$* p<0,05$

\section{Discussão}

Um pouco mais da metade das mulheres $(62,5 \%)$ realizaram a coleta de exame de $\mathrm{CV}$ durante os primeiros três meses de vida do bebê. Em consonância com a literatura (Adams et al., 2015; Buchberg et al., 2015), os resultados do presente estudo alertam para o risco de um percentual elevado de mulheres descontinuarem o próprio tratamento já no período imediato após o parto. Isso pode aumentar o risco de mortalidade dessas mulheres (Mugavero et al., 2014), de aumento significativo de CV (> 1.000 cópias/ml) e de diminuição da contagem 
de TCD4 (Aebi-Popp et al., 2016), merecendo atenção por parte dos gestores e profissionais de saúde.

Quanto às variáveis estudadas, os resultados apontaram que a contagem de TCD4 durante a gestação pode estar associada à continuidade do tratamento. Corroborando os resultados encontrados por Tariq et al. (2016), as mulheres que apresentaram maior contagem de TCD4 $\left(\geq 350\right.$ células $\left./ \mathrm{mm}^{3}\right)$ mais frequentemente não realizaram a coleta de TCD4 e/ou $\mathrm{CV}$ após o parto. Tal resultado sugere que aquelas com melhores condições clínicas tendem a abandonar mais frequentemente o próprio tratamento. Como discutido por Siddiqui, Bell, Sangi-Haghpeykar, Minard e Levison (2014), diferentemente da mulher grávida que se motiva a cuidar de sua saúde pela responsabilidade com o bebê, no momento pós-parto, a continuidade do próprio cuidado dependerá da noção de autocuidado da mulher. De fato, o estudo de Kempf et al. (2010) demonstrou que a preocupação com a própria saúde e as condições de saúde da mulher (como, por exemplo, o surgimento de outros problemas de saúde) motivavam as mulheres a comparecerem em suas consultas médicas. Nesse sentido, seria esperado que aquelas que já se encontram em um estágio avançado do HIV, AIDS, possam estar mais preocupadas com sua saúde, incentivando-as a dar seguimento aos cuidados com sua saúde, em comparação àquelas que se encontram assintomáticas e com contagem de TCD4 > 350 células $/ \mathrm{mm}^{3}$, como evidenciado no presente estudo.

Com relação aos aspectos psicossociais investigados, os resultados do presente estudo indicaram que um dos aspectos que parece influenciar a continuidade do tratamento entre as mulheres após o parto é o apoio instrumental percebido durante os primeiros três meses após o parto. Outros autores têm demonstrado que uma rede de apoio social escassa limita as possibilidades das mulheres com HIV de obter auxílio prático de outras pessoas nas diversas tarefas que demandam o cuidado de um bebê, de outros filhos e do próprio tratamento (Buchberg et al., 2015).

De fato, independentemente do contexto, o apoio social, principalmente nos primeiros meses de vida do bebê, tem sido descrito por diversos estudiosos das primeiras relações mãe-bebê como fundamental para que as mulheres consigam dedicar-se à criança, na medida em que proporcionará uma proteção física a elas, tanto em relação às exigências da realidade externa, como provendo suas necessidades vitais (Stern, 1995; Winnicott, 1960/1983).

No caso de mulheres com HIV, além da dedicação aos cuidados com o bebê, elas necessitam lidar com o próprio diagnóstico e tratamento e as preocupações com a saúde do bebê - que recebe TARV no primeiro mês de vida - que muitas vezes estão bastante intensificadas pelo medo de infectá-lo. Por todos esses aspectos, não surpreende que, nesse contexto, o apoio após o parto possa contribuir para o cuidado da mulher com a própria saúde.

No caso de mulheres com maior número de filhos, que lidam com múltiplas tarefas, como buscar outros filhos na escola e comparecer nas consultas do bebê, seguir o próprio tratamento poderá envolver maiores dificuldades. Dessa forma, certamente, para essas mulheres com mais filhos, o apoio instrumental talvez seja ainda mais relevante que continuem seu tratamento. Além disso, por toda a relevância do apoio emocional nesse momento de vida, sugere-se que outros pesquisadores investiguem sua influência na continuidade do tratamento em mulheres no pós-parto.

Por fim, destaca-se que a QV das mulheres durante a gestação pode também influenciar a continuidade do tratamento das mulheres após o parto. Dentre os domínios do WHOQoL-HIV-Bref, o do Ambiente apresentou escores mais elevados entre as mulheres que realizaram exame após o parto. Tal resultado poderia ser explicado pela relação entre QV e condições socioeconômicas (Gaspar et al., 2011; Seidl \& Tróccoli, 2006; Zimpel \& Fleck, 2007). Esse domínio parece ser mais influenciado pela ocupação, escolaridade e renda das pessoas que vivem com HIV em comparação a outros domínios também avaliados no WHOQoL-HIV-Bref (Gaspar et al., 2011). Especificamente, entre as mulheres, no estudo de Gaspar et al. (2011), aquelas com menor escolaridade e renda apresentaram menores escores nesse domínio de QV. Apesar de renda não ter sido avaliada no presente estudo, pode-se hipotetizar que a situação socioeconômica das participantes poderia estar influenciando a QV, que, por sua vez, está favorecendo que continuem o tratamento após o parto. Considerando os resultados encontrados, sugere-se que a relação entre as condições socioeconômicas, QV e continuidade do tratamento seja investigada em futuros estudos.

Já, quanto à adesão nos três meses após o parto, metade das mulheres do presente estudo (com indicação de seguir TARV e coleta de CV após o parto) apresentou CV indetectável ( $\leq 50$ cópias $/ \mathrm{ml}$ ), percentual menor do que durante a gestação, quando $62 \%$ delas apresentaram CV indetectável. Resultados similares têm sido descritos por diversos pesquisadores (Henegar et al., 2015; Kreitchmann et al., 2012; Nachega et al., 2012). 
Um dos fatores que poderia explicar o baixo percentual de adesão entre as mulheres no pós-parto é que, nesse momento de vida, como já discutido, a preocupação da mulher está dirigida para a saúde do bebê (Faria \& Piccinini, 2015; Nachega et al., 2012). Na pesquisa de Faria e Piccinini (2015), que enfocou a maternidade nesse contexto do HIV, especialmente entre as mulheres que negavam os riscos do diagnóstico para a própria saúde, a preocupação principal era cuidar do bebê pelo medo da transmissão do vírus. Tanto que geralmente conseguiam preocupar-se mais com sua saúde somente após os resultados dos primeiros exames do bebê realizados durante o primeiro ano de vida. Mais ainda, o foco dos profissionais de saúde durante a gestação e até mesmo após o parto geralmente também é evitar a transmissão vertical. Contudo, a gestação pode ser um momento oportuno para aconselhamento e apoio emocional das mulheres com HIV, favorecendo que elas continuem o próprio tratamento após o parto.

Outro aspecto que parece dificultar a adesão das mulheres após o parto é a situação atual de trabalho. As análises do presente estudo indicaram que as mulheres que trabalhavam aderiram à medicação antirretroviral mais frequentemente do que as que não trabalhavam. Talvez, esse resultado possa estar relacionado às características socioeconômicas dessas mulheres, na medida em que parece haver uma relação entre qualidade de vida, ocupação e renda. Por exemplo, como mencionado acima, o estudo de Gaspar et al. (2011), ao estudar mulheres vivendo com HIV, identificou que a ocupação e a renda das mulheres influenciavam o domínio do Ambiente da QV, que engloba a situação financeira, o acesso aos serviços de saúde e as condições de moradia, transporte e lazer. De fato, aquelas com nível socioeconômico baixo normalmente apresentam pior adesão (El-Khatib et al., 2011). Como evidenciado por Tariq et al. (2016), por exemplo, mulheres negras e migrantes costumam encontrar maiores desafios para continuidade do próprio tratamento por vivenciarem situações de pobreza, isolamento social e dificuldades de acesso à medicação. Embora o estudo de Tariq et al. (2016) tenha sido desenvolvido em outra realidade, no Reino Unido, tal achado destaca o quanto é fundamental considerar as condições de vida das mulheres ao avaliar a adesão, tendo em vista sua influência no acesso aos serviços de saúde. Dessa forma, talvez a condição de trabalho das mulheres que participaram do presente estudo reflita a condição de vida e renda delas, facilitando ou não o acesso ao serviço de saúde e, por consequência, à medicação.
Já, quanto às características clínicas das participantes, os resultados encontrados indicaram que as mulheres com CV indetectável no último trimestre da gestação mais frequentemente aderiram à TARV após o parto, apoiando uma das expectativas iniciais do presente estudo. Similarmente, outros pesquisadores têm encontrado que a supressão viral durante a gestação é um importante preditor da adesão após o parto (Mellins et al., 2008). Possivelmente, como evidenciado pela literatura, as mulheres com CV indetectável na gravidez geralmente são aquelas que iniciaram mais precocemente o pré-natal e com maior número de consultas realizadas na gestação (Faria et al., 2014). O início precoce do pré-natal provavelmente indica o cuidado e a preocupação dessas gestantes com seu bebê e sua saúde, motivando-as para o uso correto de TARV.

Ainda quanto às características clínicas, outro resultado que merece destaque é que as mulheres com adesão no pós-parto apresentaram significativamente maior contagem de TCD4 do que aquelas que não aderiram à medicação. Tal resultado seria esperado, na medida em que o uso correto da medicação antirretroviral restaura parcialmente o sistema imunológico das pessoas que vivem com HIV (Deeks et al., 2013; Grinsztejn et al., 2014; Lundgren et al., 2015). Por sua vez, como tem sido demonstrado por esses pesquisadores, isso leva à redução das complicações relacionadas à AIDS, o que é de extrema importância para a saúde e aumento da sobrevida dessas mães.

Já, no que se refere aos aspectos psicossociais investigados, diferentemente da expectativa inicial, não se encontrou menor adesão entre mulheres com depressão. Talvez o BDI não tenha sido o instrumento mais apropriado para avaliar depressão em mulheres no contexto do HIV após o parto, dificultando identificar mulheres com ou sem depressão. Nesse momento de vida, comumente tem sido utilizada a Escala de Depressão Pós-Parto de Edinburgo (EPDS), que foi desenvolvida para triagem de depressão pós-parto, evitando confundir sintomas somáticos da depressão e queixas comuns em mães no pós-parto (Stringer et al., 2014). Do mesmo modo, entre mulheres com HIV, especialmente em estágio avançado, como muitas das participantes deste estudo, o BDI pode superestimar a presença de sintomas depressivos.

\section{Considerações Finais}

Em conjunto, os resultados do presente estudo enfatizam a relevância da satisfação e disponibilidade 
de apoio social para a continuidade do tratamento em mulheres que vivem com HIV durante os primeiros três meses de vida do bebê. Além disso, ficou evidente a importância das condições de vida e da própria situação clínica dessas mulheres, que poderão ou não facilitar a continuidade do tratamento e a adesão após o parto. Levando em conta tais achados, destaca-se a relevância do apoio social, na medida em que podem contribuir para o cuidado delas com sua saúde após nascimento do bebê, quando elas, a família e o serviço de saúde tendem a dedicar-se prioritariamente ao bebê. Do mesmo modo, sugere-se que sejam implementadas intervenções que facilitem o acesso das mulheres aos serviços de saúde, em particular para aquelas com piores condições socioeconômicas.

Por fim, cabe destacar algumas limitações do presente estudo. Uma das limitações é o baixo poder de generalização dos achados, seja em função do número de participantes, seja ao se limitar a mulheres com características clínicas e sociodemográficas similares. As mulheres que não participaram das coletas de dados da Fase II ( $3^{\circ}$ mês) eram menos escolarizadas e com início mais tardio do pré-natal. Dessa forma, as participantes do presente estudo, mais escolarizadas, mesmo que fossem bastante pobres e socialmente vulneráveis, ainda assim apresentavam melhores condições de vida do que aquelas que não seguiram participando da pesquisa, o que deveria ser considerado ao analisar os achados do presente estudo. Além disso, o tamanho da amostra não permitiu outras análises estatísticas, como regressão múltipla ou logística, que possibilitaria identificar fatores de risco para descontinuidade do tratamento de HIV em mulheres após o parto.

\section{Referências}

Adams, J. W., Brady, K. A., Michael, Y. L., Yehia, B. R., \& Momplaisir, F. M. (2015). Postpartum engagement in HIV care: An important predictor of long-term retention in care and viral suppression. Clinical Infectious Diseases, 61(12), 1880-1887. doi: 10.1093/cid/civ678

Aebi-Popp, K., Kouyos, R., Bertisch, B., Staehelin, C., Rudin, C., Hoesli, I., ... Yerly, S. (2016). Postnatal retention in HIV care: Insight from the Swiss HIV cohort study over a 15 -year observational period. HIV Medicine, 17(4), 280-288. doi: 10.1111/ hiv.12299
Buchberg, M. K., Fletcher, F. E., Vidrine, D. J., Levison, J., Peters, M. Y., Hardwicke, R., ... Bell, T. K. (2015). A mixed-methods approach to understanding barriers to postpartum retention in care among low-income, HIV-infected women. AIDS Patient Care and STDs, 29(3), 126-132. doi: 10.1089/ apc. 2014.0227

Carvalho, F. T. (2009). Contribuições de uma intervenção psicoeducativa para estratégias de enfrentamento e qualidade de vida de gestantes portadoras de HIV / AIDS (Tese de doutorado) Universidade Federal do Rio Grande do Sul. Recuperado de https://www.lume.ufrgs. br/bitstream/handle/10183/17212/000707695. pdf?sequence $=1$

Cohen, M. S., Chen, Y. Q., McCauley, M., Gamble, T., Hosseinipour, M. C., Kumarasamy, N., ... Fleming, T. R. (2011). Prevention of HIV-1 infection with early antiretroviral therapy. The New England Journal of Medicine, 365(6), 493-505. doi: 10.1056/ NEJMoa1105243

Cunha, J. A. (2001). Escalas Beck. São Paulo: Casa do Psicólogo.

Deeks, S. G., Lewin, S. R., \& Havlir, D. V. (2013). The end of AIDS: HIV infection as a chronic disease. Lancet, 382(9903),1525-1533. doi: 10.1016/ S0140-6736(13)61809-7

El-Khatib, Z., Ekstrom, A. M., Coovadia, A., Abrams, E. J., Petzold, M., Katzenstein, D., ... Kuhn, L. (2011). Adherence and virologic suppression during the first 24 weeks on antiretroviral therapy among women in Johannesburg, South Africa - A prospective cohort study. BMC Public Health, 11(1), 88. doi: 10.1186/1471-2458-11-88

Faria, E. R., Carvalho, F. T., Lopes, R. S., Piccinini, C. A., Gonçalves, T. R., \& Santos, B. R. (2014). Gestação e HIV: Preditores da adesão ao tratamento no con texto do pré-natal. Psicologia: Teoria e Pesquisa, 30(2), 197-203. doi: 10.1590/ S0102-37722014000200009

Faria, E. R., Gonçalves, T. R., Carvalho, F. T., Lopes, R. C. S., \& Piccinini, C. A. (2014). Coping strategies among brazilian pregnant women living with HIV. Paidéia, 24(57), 67-74. doi: 10.3329/bmrcb. v37i1.7791

Faria, E. R., \& Piccinini, C. A. (2015). Representações maternas no contexto do HIV: Gestação ao 
segundo ano da criança. Psicologia em Estudo, Maringá, 20(4), 625-637. doi: 10.4025/psicolestud. v20i4.28749

Gaspar, J., Reis, R. K., Pereira, F. M. V., Grinsztejn, B., Hosseinipour, M. C., Ribaudo, H. J., Swindells, S., Eron, J., Chen, Y. Q., ... Cohen, M. S. (2014). Effects of early versus delayed initiation of antiretroviral treatment on clinical outcomes of HIV-1 infection: Results from the phase 3 HPTN 052 randomised controlled trial. The Lancet Infectious Diseases, 14(4), 281-290. doi: 10.1016/S1473-3099(13)70692-3

Grinsztejn, B., Hosseinipour, M. C., Ribaudo, H. J., Swindells, S., Eron, J., Chen, Y. Q., ... Cohen, M. S. (2014). Effects of early versus delayed initiation of antiretroviral treatment on clinical outcomes of HIV-1 infection: Results from the phase 3 HPTN 052 randomised controlled trial. The Lancet Infectious Diseases, 14(4), 281-290. http://doi. org/10.1016/S1473-3099(13)70692-3

Gugsa, S., Potter, K., Tweya, H., Phiri, S., Sande, O., Sikwese, P., ... O’Malley, G. (2017). Exploring factors associated with ART adherence and retention in care under Option B+ strategy in Malawi: A qualitative study. PLOS ONE, 12(6), 1-18. doi: 10.1371/journal.pone.0179838

Henegar, C. E., Westreich, D. J., Maskew, M., Miller, W. C., Brookhart, M. A., \& Rie, A. Van. (2015). Effect of pregnancy and the postpartum period on adherence to antiretroviral therapy among HIVInfected women established on treatment. Journal of Acquired Immune Deficiency Syndromes, 68(4), 477480. doi: 10.1097/QAI.0000000000000501

Hodgson, I., Plummer, M. L., Konopka, S. N., Colvin, C. J., Jonas, E., Albertini, J., ... Fogg, K. P. (2014). A systematic review of individual and contextual factors affecting ART initiation, adherence, and retention for HIV-infected pregnant and postpartum women. PloS one, 9(11), e111421. doi:10.1371/ journal.pone.0111421

Kay, E. S., Batey, D. S., \& Mugavero, M. J. (2016). The HIV treatment cascade and care continuum: Updates, goals, and recommendations for the future. AIDS Research and Therapy, 13(1). doi:10.1186/ s12981-016-0120-0

Kempf, M.-C., McLeod, J., Boehme, A. K., Walcott, M. W., Wright, L., Seal, P., .. Moneyham, L. (2010). A qualitative study of the barriers and facilitators to retention-in-care among HIV-positive women in the rural southeastern United States: Implications for targeted interventions. AIDS Patient Care and STDs, 24(8), 515-520. doi:10.1089/apc.2010.0065

Kreitchmann, R., Harris, D. R., Kakehasi, F., Haberer, J. E., Cahn, P., Losso, M., ... Read, J. S. (2012). Antiretroviral adherence during pregnancy and postpartum in Latin America. AIDS Patient Care and STDs, 26(8), 486-95. doi:10.1089/apc.2012.0013

Lundgren, J. D., Babiker, A. G., Gordin, F., Emery, S., Grund, B., Sharma, S., ... Neaton, J. D. (2015). Initiation of Antiretroviral Therapy in Early Asymptomatic HIV Infection. New England Journal of Medicine, 373(9), 795-807. doi:10.1056/ NEJMoa1506816

Mellins, C. A., Chu, C., Malee, K., Allison, S., Smith, R., Harris, L., ... Larussa, P. (2008). Adherence to antiretroviral treatment among pregnant and postpartum HIV-infected women. AIDS Care, 20(8), 958-968. doi:10.1080/09540120701767208

Momplaisir, F. M., Storm, D. S., Nkwihoreze, H., Jayeola, O., \& Jemmott, J. B. (2018). Improving postpartum retention in care for women living with HIV in the United States. AIDS (London, England), 32(2), 133142. doi:10.1097/QAD.0000000000001707

Mugavero, M. J., Westfall, A. O., Cole, S. R., Geng, E. H., Crane, H. M., Kitahata, M. M., ... Raper, J. L. (2014). Beyond core indicators of retention in HIV care: Missed clinic visits are independently associated with all-cause mortality. Clinical Infectious Diseases, 59(10), 1471-1479. doi:10.1093/cid/ ciu603

Mugavero, M. J., Westfall, A. O., Zinski, A., Drainoni, M., Gardner, L. I., Keruly, J. C., ... Wilson, T. E. (2012). Measuring retention in HIV care: The elusive gold standard. Journal of Acquired Immune Deficiency Syndromes, 61(5), 574580. doi:10.1097/ QAI.0b013e318273762f.Measuring

Nachega, J. B., Uthman, O. A., Anderson, J., Peltzer, K., Wampold, S., Cotton, M. F., ... Mofenson, L. M. (2012). Adherence to antiretroviral therapy during and after pregnancy in low-income, middleincome, and high-income countries. AIDS, 26(16), 2039-2052. doi:10.1097/QAD.0b013e328359590f

Ngarina, M., Popenoe, R., Kilewo, C., Biberfeld, G., \& Ekstrom, A. M. (2013). Reasons for poor adherence to antiretroviral therapy postnatally in 
HIV-1 infected women treated for their own health: experiences from the Mitra Plus study in Tanzania. BMC Public Health, 13, 450. doi:10.1186/1471-2458-13-450

Phillips, T., Thebus, E., Bekker, L.-G., Mcintyre, J., Abrams, E. J., \& Myer, L. (2014). Disengagement of HIV-positive pregnant and postpartum women from antiretroviral therapy services: a cohort study. Journal of the International AIDS Society, 17(1), 19242. doi:10.7448/IAS.17.1.19242

Robson, C. \& McCartan, K. (2016). Real World Research: A Resource for Users of Social Research Methods um Applied Settings. Nova Jersey: Wiley.

Seidl, E. M. F., \& Tróccoli, B. T. (2006). Desenvolvimento de escala para avaliação do suporte social em HIV/Aids. Psicologia: Teoria e Pesquisa, 22(3), 317-326. doi:10.1590/S0102-37722006000300008

Siddiqui, R., Bell, T., Sangi-Haghpeykar, H., Minard, C., \& Levison, J. (2014). Predictive factors for loss to postpartum follow-up among low income HIVInfected women in Texas. AIDS Patient Care and STDs, 28(5), 248-253. doi:10.1089/apc.2013.0321

Stern, D. N. (1995). The motherhood constellation: A unified view of parent-infant psychotherapy. New York: BasicBooks.

Stringer, E. M., Meltzer-Brody, S., Kasaro, M., Stuebe, A. M., Wiegand, S., Paul, R., \& Stringer, J. S. A. (2014). Depression, pregnancy, and HIV: The case to strengthen mental health services for pregnant and post-partum women in Sub-Saharan Africa. The Lancet Psychiatry, 1(2), 159-162. doi:10.1016/ S2215-0366(14)70273-1

Swain, C.-A., Smith, L. C., Nash, D., Pulver, W. P., Gordon, D., Bian, F., ... McNutt, L.-A. (2016). Postpartum human immunodeficiency virus care among women diagnosed during pregnancy. $\mathrm{Ob}$ stetrics \& Gynecology, 128(1), 44-51. doi:10.1097/ AOG.0000000000001454
Tariq, S., Elford, J., Chau, C., French, C., Cortina-Borja, M., Brown, A., ... Tookey, P. A. (2016). Loss to follow-up after pregnancy among Sub-Saharan Africa-born women living with human immunodeficiency virus in England, Wales and Northern Ireland: results from a large national cohort. Sexually Transmitted Diseases, 43(5), 283-289. doi:10.1097/ OLQ.0000000000000442

Wang, K., Chen, W. T., Lin, Z., Bao, M. J., Zhao, H., \& Lu, H. (2016). Facilitators of and barriers to HIV self-management: Perspectives of HIV-positive women in China. Applied Nursing Research, 32, 9197. doi:10.1016/j.apnr.2016.06.004

Winnicott, D. W. (1983). Teoria do relacionamento paterno-filial. Em D. W. Winnicott (Ed.), O Ambiente e os processos de maturação (pp. 38-54). Porto Alegre: ArtMed.

World Health Organization - WHO. (2013). Consolidated guidelines on the use of antiretroviral drugs for treating and preventing hiv infection. World Health Organization. Geneva: WHO.

World Health Organization. (2015). Consolidated Strategic Information Guidelines for HIV in the Health Sector. Geneva: World Health Organization. Recuperado de https://apps.who.int/iris/bitstream/handle/1 0665/164716/9789241508759_eng.pdf;jsessionid $=15 \mathrm{CFA} 8 \mathrm{AC} 2 \mathrm{FB} 1 \mathrm{E} 01 \mathrm{CA} 1970578151953 \mathrm{C} 6$ ?seq uence $=1$

Zimpel,R.R.,\&Fleck, M.P. (2007). Quality of life in HIV-positive Brazilians: Application and validation of the WHOQOL-HIV, Brazilian version. AIDS Care - Psychological and Socio-Medical Aspects of AIDS / HIV, 19(7), 923-930. doi:10.1080/09540120701213765

Recebido em: 20/03/2019 Reformulado em: 28/04/2020 Aprovado em: 13/05/2020 
Sobre os autores:

Fernanda Borges de Medeiros é graduada em Psicologia pela Universidade Federal do Rio Grande do Sul (UFRGS) e especialista em Psicologia Hospitalar pela Faculdade de Medicina da Universidade de São Paulo (FMUSP). É mestre e doutora em Psicologia pela UFRGS.

ORCID: https://orcid.org/0000-0001-6333-1147

E-mail: fernanda.bdmedeiros@gmail.com

Evelise Rigoni de Faria é psicóloga do Grupo Hospitalar Conceição (GHC/RS) e docente do Programa de Pós-Graduação em Avaliação de Tecnologias para o SUS (PPG ATS-SUS GHC). É mestre e doutora em Psicologia pela Universidade Federal do Rio Grande do Sul (UFRGS).

ORCID: https://orcid.org/0000-0003-0053-6350

E-mail: everigoni@gmail.com

Cesar Augusto Piccinini é doutor e pós-doutor pela University College London (Inglaterra). É professor titular do Instituto de Psicologia da Universidade Federal do Rio Grande do Sul (UFRGS) e coordenador do Núcleo de Infância e Família (NUDIF) da UFRGS.

ORCID: https://orcid.org/0000-0002-4313-3247

E-mail: piccicesar@gmail.com

Contato com os autores:

Ramiro Barcelos, 2600, sala 111

Porto Alegre-RS, Brasil

CEP: 90035-003 